\title{
DETERMINAÇÃO DE HIDRATAÇÃO, OLEOSIDADE E PH FACIAL DE USUÁRIAS E NÃO USUÁRIAS DE MAQUIAGENS
}

\section{ARTIGO ORIGINAL}

SANTOS, Aldeane da Silva ${ }^{1}$

SILVA, Aryane Lúcia da ${ }^{2}$

VASCONCELOS, Tibério de Lima ${ }^{3}$

SANTOS, Aldeane da Silva. SILVA, Aryane Lúcia da. VASCONCELOS, Tibério de Lima. Determinação de hidratação, oleosidade e Ph facial de usuárias e não usuárias de maquiagens. Revista Científica Multidisciplinar Núcleo do Conhecimento. Ano 06, Ed. 01, Vol. 03, pp. 186-219. Janeiro de 2021. ISSN: 24480959, Link de acesso: https://www.nucleodoconhecimento.com.br/saude/hidratacaooleosidade

\section{RESUMO}

A pele é o maior órgão do corpo humano e atua formando uma barreira protetora, controlando a passagem de água, eletrólitos e a termorregulação do corpo. A oleosidade e a hidratação superficial da pele são fatores essenciais para a manutenção da sua homeostasia. As bases corretivas são produtos destinados a cobrir a região do rosto fornecendo coloração uniforme para aplicação de outros pigmentos colorantes. $\mathrm{O}$ objetivo deste trabalho foi analisar comparativamente a oleosidade, a hidratação e o pH facial da pele de mulheres usuárias e não usuárias de base corretiva. $O$ estudo realizado foi de caráter exploratório quantitativo de campo,

\footnotetext{
${ }^{1}$ Bacharel em Farmácia.

${ }^{2}$ Bacharel em Farmácia.

${ }^{3}$ Orientador. Farmacêutico, Mestre em Química pela UFRPE, MBA em Cosmetologia, Graduado em Farmácia pela ASCES.
} 
realizado em 58 mulheres com idade entre 18 e 35 anos, aplicando-se o questionário e empregando-se o equipamento de análise facial por bioimpedância e o equipamento pHmetro com sonda superficial. As medidas foram realizadas em triplicata na região masseteriana. De acordo com o questionário o tipo de base corretiva mais utilizada pelas entrevistadas foi à base líquida, os agentes de limpeza mais empregados na rotina de higienização da pele foram água e sabão e os problemas de pele mais prevalentes foram acne e alergias. Não houve diferenças significativas na comparação dos valores médios de oleosidade, hidratação e pH das usuárias e não usuárias de base corretiva. Conclui-se com a pesquisa que o uso de base corretiva não interferiu nos parâmetros analisados.

Palavras-Chave: Pele, cosméticos, hidratação.

\section{INTRODUÇÃO}

A pele é o maior órgão do corpo humano e atua formando uma barreira física protetora, controlando a passagem de água e eletrólitos e a termorregulação do corpo. É um órgão complexo com diferentes tipos de células, e que apresenta alterações diárias em seus parâmetros como temperatura, produção de sebo, pH, hidratação, e perda de água transepidérmica (TEWL). Os seus parâmetros biofísicos sofrem variações diárias devido à exposição à radiação ultravioleta e às temperaturas (FIROOZ, 2016).

A pele divide-se em três camadas: epiderme, derme e tecido subcutâneo. As células epidérmicas são os primeiros queratinócitos, que migram para superfície da pele durante a maturação formando o estrato córneo, sendo constituído de epitélio estratificado, composto de estruturas lipídicas intercelulares, como ceramidas, colesterol e ácidos graxos, que atuam como uma barreira contra a desidratação. As mudanças na pele ocorrem devido aos fatores intrínsecos e extrínsecos que podem levar ao envelhecimento precoce, alterações bioquímicas, celulares e teciduais resultando na diminuição da ligação dermo-epiderme, essas alterações prejudicam o transporte de nutrientes entre as camadas da pele causando diminuição da matriz extracelular e a fragmentação das fibras elásticas, consequentemente ocorrerá 
diminuição da síntese de colágeno prejudicando a atividade dos fibroblastos. Pesquisas apontam que as substâncias e os componentes ativos presentes nas formulações podem influenciar nas propriedades mecânicas da pele (RANGEL et al., 2014).

O mercado de cosméticos mundial apresenta uma grande diversidade de produtos e constantemente novas matérias primas são lançadas para que o processo de inovação no seguimento seja cada vez mais intenso (MONTEIRO, 2017). As maquiagens são uma das categorias de produtos mais consumidas por mulheres, com o surgimento de ativos com efeito protetor esses produtos passaram a desempenhar não somente o efeito embelezador, mas também o de proteger, tratar e promover benefícios de longo prazo (IGWO-EZIKPE, 2017).

Com uso cada vez mais frequente, esses produtos vêm ocupando espaço nos cuidados diários com a pele, e sendo este o maior órgão do corpo humano, os cuidados são imprescindíveis para que se possam manter as condições fisiológicas em perfeito funcionamento. Um dos principais fatores para a manutenção da homeostasia cutânea é a oleosidade e hidratação superficial da pele. A oleosidade permite a manutenção e o aumento do nível de hidratação profunda da pele, evitando a perda hídrica por evaporação, logo a hidratação mantida na pele promove o fluxo de processos bioquímicos naturais que resultam numa pele saudável e com aspecto saudável (LUEBBERDING, 2013).

As bases corretivas são produtos indicados para cobrir a região do rosto, formando um filme de característica lipofílica contendo pigmentos tonalizantes que mimetizam a coloração natural da pele com objetivo de uniformizar e fornecer uma área com coloração uniforme para aplicação de outros pigmentos colorantes. Entretanto, hoje, com surgimento de novas matérias primas, esses produtos tiveram suas funções potencializadas e passam a fazer parte de outra categoria denominada comercialmente como cosméticos multifuncionais, os BB creams (Cremes BlemishBalm), CC creams (cremes Color Correction) e DD creams (cremes Daily Defense) (SCHNITTGER, 2007). 
Recentemente os aparelhos de avaliação de oleosidade e hidratação que usam sensores de bioimpedância passaram a serem popularizados, trazendo uma ferramenta útil para pesquisas e análises diárias desses fatores. As publicações utilizando esses equipamentos passaram a ganhar espaço nos periódicos científicos pela facilidade no manuseio, precisão das medidas, baixo custo e resultados rápidos (CONSTANTIN, 2014).

Desta forma, objetiva-se nesse estudo determinar a oleosidade, hidratação e pH superficial da pele de mulheres que utilizam base corretiva diariamente e de mulheres que não tem esse hábito, a frequência do uso de produtos cosméticos, a utilização de removedores de maquiagens e a idade da entrevistada.

\section{REFERÊNCIAL TEÓRICO}

\section{FATORES DE HIDRATAÇÃO, OLEOSIDADE NATURAL DA PELE E PH}

A água é o principal componente de células e tecidos do corpo humano, e desempenha um papel fundamental no equilíbrio fisiológico normal. Representa $75 \%$ da composição corporal, possui nutrientes e propriedades essenciais que atuam na homeostasia celular, além de auxiliar na manutenção do volume corporal (intracelular e extracelular). A hidratação da pele ajuda na termorregulação do corpo e funciona como um lubrificante e absorvente de choque. Pesquisas apontam que a água produzida pelo metabolismo celular e a água fornecida por alimentos e bebidas, não é suficiente para atender às necessidades diárias de água do corpo. Quanto à fisiologia da pele humana, o conteúdo de água é importante para as diferentes funções da pele, como a função "barreira" da água ou a função "envelope", e a deficiência hídrica que está relacionada às disfunções dermatológicas. A ingestão de água pode ser benéfica para a saúde, principalmente em termos dermatológicos (antienvelhecimento). Contudo, a relação direta entre as propriedades e o consumo regular de água na dieta não é bem esclarecido devido a poucas publicações (LUEBBERDING, 2013). 
As propriedades fisiológicas da pele variam entre homens e mulheres porque as necessidades terapêuticas de ambos em relação à pele são frequentemente diversas, essas diferenças inclui a perda de água transepidérmica (TEWL), hidratação do extrato córneo (SC hidratação), conteúdo de sebo, valor de $\mathrm{pH}$, produção de hormônio e o surgimento de várias doenças como acne rosácea e eczema seborreico. Embora algumas pesquisas revelem que há uma maior produção de TWEL e sebo em homens quando comparado às mulheres, ao que tudo indica, não há entendimento que o gênero influência nas propriedades fisiológicas da pele. A hidratação da pele foi avaliada por métodos de medição biofísicos usados mundialmente (LUEBBERDING, 2013)

A oleosidade da pele é comum em homens e mulheres e frequentemente ocorre antes da puberdade. A pele oleosa pode estar associada aos produtos cosméticos, aos fatores externos e internos que podem influenciar no surgimento de acne. A oleosidade da pele causada por produtos cosméticos é uma consequência das glândulas sebáceas que produzem uma quantidade maior de sebo dando aparência de brilho e pele oleosa. Pode estar associada a dieta, idade, sexo, etnia, climas quentes e úmidos. A área do corpo mais afetada pela oleosidade é o rosto devido a grande quantidade de glândulas sebáceas. Normalmente a aparência oleosa se encontra na zona $\mathrm{T}$ (testa, nariz e queixo) onde se remete maior predomínio das glândulas sebáceas. A avaliação da oleosidade da pele pode ser medida precisamente através dos métodos não invasivos, como almofadas de papel absorvente, avaliação fotométrica (por exemplo, SubmeterSM 810; CK electronic), argila de bentonita e fitas sensíveis a lipídios (por exemplo ,Sebutape; CuDermCorp.). Porém, os fatores ambientais e biológicos podem influenciar nos resultados, sendo necessários estudos metodológicos mais rigorosos. (SAKUMA, 2012).

$\mathrm{O}$ pH na superfície da pele é importante para defesa antimicrobiana e regulação da atividade de enzima e expressão epidérmica. $\mathrm{O}$ pH também influencia no tamponamento da pele que sofre efeitos internos e externos tanto em relação à acidificação como à alcalinização. Os homens apresentam valores de $\mathrm{pH}$ menores que as mulheres independentemente da localização e idade do indivíduo. O que 
podem influenciar a acidificação do pH da superfície da pele são os ácidos láctico, ácidos graxos livres, gerado pelas lipases das glândulas sebáceas e pelas glândulas écrinas. O medidor de pH da pele PHmetria PH 905 (Courage \& Khazaka) mede diretamente a película ácida hidrofílica da superfície da pele. A medição do nível de pH é um parâmetro importante para analisar a qualidade do filme hidro-lipídeo na superfície da pele. (MATSUMOTO, 2013).

$\mathrm{O} \mathrm{pH}$ da pele e o índice de cor da pele sofrem variações no decorrer do dia, essas variações nos parâmetros da pele devem ser consideradas para pesquisas futuras, podem ter significado clínico para a seleção de produtos para o cuidado da pele (FIROOZ, 2016).

\section{DERMATITES E ALERGIAS COSMÉTICAS}

A indústria de cosméticos nos últimos anos vem crescendo em ritmo acelerado, com a aplicação de novas tecnologias empregadas nos produtos cosméticos que apresentam uma variedade de matérias-primas destinadas à higiene pessoal e à beleza e que frequentemente apresentam uma vasta lista de ingredientes que vão de ativos aos excipientes (MONTEIRO 2017). É importante entender como esses produtos podem interagir com a pele, a aderência sobre a pele e quais benefícios causados à pele, bem como é imprescindível compreender a mecânica da pele e a interação cosmético e pele, para obter recursos visuais e efeitos funcionais para se ter produtos seguros e eficazes. Com a diversidade de matérias-primas utilizadas em cosméticos que oferecem hidratação ou redução dos sinais do envelhecimento, proteção as agressões diárias, esses produtos devem ser eficazes, seguros, biodisponíveis e inovadores. (SCHNITTGER, 2007).

Os produtos cosméticos são utilizados diariamente por milhões de consumidores tanto por homens como por mulheres, são aplicados sobre a pele humana para limpeza do corpo, embelezamento, promoção, atratividade ou para alterar a aparência sem afetar a estrutura ou funções do corpo. Apesar de a pele possuir uma barreira protetora, as substâncias podem penetrar nas cavidades e mucosas produzindo efeito local e sistêmico, como irritação, sensibilização, alergia ou fotorreações (NAZZARO, 2016). 
Os cosméticos são compostos de produtos naturais ou sintéticos produzidos através da mistura de produtos químicos, como metais pesados e policíclicos hidrocarbonetos (PAHs). Os metais tóxicos encontrados em cosméticos são chumbo, arsênico, níquel, mercúrio, cádmio e cromo, esses constituintes são encontrados naturalmente no meio ambiente, nas rochas, solo, água e ar, portanto, podem ser encontrados em pigmentos e outras matérias-primas usadas na indústria de cosméticos. A utilização dessas substâncias em cosméticos pode estar atribuída ao uso intencional durante a fabricação, ou às impurezas encontradas nas matérias-primas. Essas substâncias quando excedem as concentrações mínimas promovem estresse oxidativo, provocando sérios riscos à saúde. Portanto, há uma grande preocupação com os produtos de cuidados pessoais para que não constem em sua formulação substâncias irritantes e que causem risco à saúde dos consumidores a curto e longo prazo. Diante dos potenciais efeitos adversos dessas substâncias presentes em formulações de maquiagem facial se faz necessário um monitoramento constante sobre a segurança desses produtos. Atualmente os cosméticos apresentam uma grande variedade de produtos para o cuidado com a pele, com diversas funções que atuam diretamente sobre a pele, como: hidratação, clareamento, proteção solar e antiacne. Essas formulações apresentam matérias- primas como dióxido de titânio, óxido de zinco para produtos com cores e pigmentos, e silicones que são utilizados para diversas atividades (IGWO-EZIKPE, 2017).

As maquiagens possuem em sua formulação diferentes pigmentos e misturas de cor. Os pigmentos convencionais são usados para gerar tons e cores, pigmentos que geram diversas cores podem ser orgânicos, inorgânicos ou são utilizados para melhorar a funcionalidade do produto e são capazes de criar ilusões de uma forma que escondem rugas e manchas, são classificados como partículas microfinas, ultrafinas ou nanométricas. Os inorgânicos consistem em titânio dióxido de carbono, óxido de zinco, óxidos de ferro e ultramarines. Pós e pigmentos podem ser modificados para tornar a superfície repelente de água (hidrofóbico), dispersível em óleo (lipofílico), ou hidrofílico (SCHNITTGER, 2007). 
As maquiagens são produtos comumente utilizados diariamente pela maioria das mulheres, a fim de encobrir imperfeições na pele, embelezar, e aumentar a autoestima do indivíduo. Na maioria das vezes as maquiagens podem causar acne vulgar, por apresentar em sua composição substâncias comedogênicas que estimulam a produção de sebo pelas glândulas sebáceas. Parte dos dermatologistas restringem a utilização de maquiagens, pois consideram a maquiagem cosmética um fator crucial para o surgimento de acne, sendo que a maioria das mulheres submetidas ao tratamento de acne continua a utilizar maquiagem mesmo com a advertência do dermatologista (MATSUOKA, 2006).

\section{MÉTODOS NÃO INVASIVOS NA ANÁLISE DA PELE}

As tecnologias não invasivas de bioengenharia desenvolvidas nas últimas décadas proporcionam um insumo significativo na dermatologia e no desenvolvimento de pesquisas, educação médica e atendimento ao paciente. Uma das tecnologias utilizadas não invasiva é o corneômetro, utilizado para medir o grau de hidratação epidérmica em condições saudáveis ou patológicas, é um método confiável, barato, de fácil utilização, que não causa danos à saúde e analisa a eficácia de algumas preparações destinadas a aplicação da superfície da pele (CONSTANTIN, 2014).

O controle da hidratação da pele é uma técnica importante na dermatologia e cosmetologia onde se verifica várias doenças e define-se a eficácia das terapias médicas e a função dos tratamentos antienvelhecimento e de hidratação. Os níveis de hidratação da pele são medidos através dos métodos de impedância elétrica da pele, condutividade térmica, técnicas de espectroscopia óptica, incluindo refletividade, e por métodos indiretos de avaliação das propriedades mecânicas da pele, ou sua geometria superficial (BENJAMIN et al., 2005). O método de impedância elétrica é o mais confiável e estável na avaliação da pele. É um método simples, com custo mínimo e que não causa prejuízo à saúde. Esse dispositivo apresenta sensores ultrafinos, elásticos, capazes de laminação conformada sobre a pele, para medição precisa e mapeamento espacial dos níveis de hidratação, apresenta ótima precisão e exatidão quando comparados aos dispositivos convencionais. O contato suave deste dispositivo sobre a pele de modo reversível e não invasivo permite o mapeamento 
espacial da hidratação em altos níveis de resolução, mediante a adição de sensores de medição e sua utilização é útil para avaliações dos parâmetros da pele (XIAN HUANG, 2012).

Vários dispositivos mecânicos desenvolvidos são capazes de detectar mudanças nas propriedades viscoelásticas da pele, como por exemplo, (cortometros, dermaflexose medidores de toque dérmico (eles usam métodos de medição diferentes). Entretanto, ainda não foi abordado um método que determina as propriedades viscoelásticas da pele com exatidão. O método de torção utiliza os medidores de toque dérmico. $O$ dermoflex é um dispositivo de câmera que utiliza o método por sucção. O cortomero é um dispositivo com métodos não invasivos que determina as propriedades viscoelásticas da pele humana através do método de sucção, usando um padrão que compara a induração da pele e o edema, sendo utilizado em pesquisa a respeito da pele normal e das doenças que atingem a pele, como psoríase, esclerodermia, erisipela, dentre outros. No entanto, os cortometros apresentam o método de sucção com as sondas com diâmetros maiores, que pode chegar à camada mais profunda da pele (derme e subcutânea) causando deformação e interferindo nos resultados. (HYU, 2008).

Frequentemente são utilizadas técnicas invasivas das propriedades biomecânicas da pele para a análise do tecido da pele in vivo. A escanometria é o método mais utilizado para pesquisa in vivo na subcamada da pele. Neste método são realizadas várias aplicações de filme adesivo, que podem causar mudanças no perfil da espessura da pele removida devido à força de padronização aplicada. Atualmente são utilizados poucos métodos não invasivos para determinação da hidratação da pele, as medições da pele têm sido conduzidas pela medição de propriedades elétricas, medidas de capacitância da pele, condutância, perda de água transpidermal (TEWL) e eletrodo de $\mathrm{pH}$. Esses métodos fornecem informações sobre as mudanças nas características gerais da pele, portanto eles não determinam as mudanças em sua composição molecular. Com as limitações das técnicas convencionais são realizadas várias pesquisas utilizando métodos não invasivos para análise da pele em tempo real, dentre essas técnicas in vivo o método de espestroscopia confocal Raman é 
excelente para identificar e caracterizar o processo de envelhecimento através dos processos bioquímico, estruturais e moleculares do tecido vivo. (RANGEL et al., 2014).

\section{METODOLOGIA}

\section{TIPO DE ESTUDO}

Trata-se de um estudo exploratório quantitativo e de campo realizado no Centro Universitário do Vale do Ipojuca - UNIFAVIP/WYDEN, sobre a análise comparativa de hidratação e pH facial superficial da pele de mulheres usuárias e não usuárias de maquiagens do tipo base corretiva . A região analisada foi a da bochecha conhecida como região masseterina mediante a aplicação dos questionários e método não invasivo.

Esta pesquisa foi realizada de acordo com a Resolução no 510/2016 do Conselho Nacional de Saúde, tendo o projeto sido aprovado pelo Comitê de Ética em pesquisa da UniFavip/Wyden sob CAAE no 99790818.6.0000.5666 e parecer oㅜ 2.972.239.

\section{LOCAL DA PESQUISA}

A pesquisa foi realizada no Centro Universitário Vale do Ipojuca (UNIFAVIP/ WYDEN), localizado na Rua Adjar da Silva Casé, 800, CEP 55024-740 - no Bairro Indianópolis, Caruaru - PE.

\section{POPULAÇÃO E AMOSTRA}

A amostragem se deu por conveniência e o público participante da pesquisa foi composto por 68 mulheres estudantes do Centro Universitário Vale do Ipojuca (UNIFAVIP/ WYDEN), com idade entre 18 e 35 anos que se encaixarem nos critérios de inclusão. 


\section{CRITÉRIO DE INCLUSÃO E CRITÉRIOS DE EXCLUSÃO}

No estudo foram incluídos os usuários capazes de se comunicar e que concordaram em responder ao conjunto de questões apresentadas e permitiram a realização das aferições mediante assinatura do Termo de Consentimento Livre e Esclarecido.

Não foi alvo deste estudo, menores de idade ou pessoas que não possam responder legalmente por suas ações, mulheres com dermatite de contato ou seborreica, acne grau 2, 3 ou 4, que tiveram exposição recente à radiação ultravioleta, grávidas, lactantes, em uso de contraceptivos orais, corticóides, ou em tratamento dermocosméticos e estéticos recentes que podem alterar os parâmetros da pele.

\section{PROCEDIMENTOS E COLETA DOS DADOS}

\section{APLICAÇÃO DO QUESTIONÁRIO}

A aplicação do questionário adaptado de KIMLIN (2012) contendo perguntas específicas relativas à caracterização das mulheres que usam e das que não fazem uso de bases corretivas se deu após concordarem em participar na pesquisa mediante assinatura do TCLE.

\section{ANÁLISE DE HIDRATAÇÃO E OLEOSIDADE FACIAL}

Em uma sala fechada com temperatura $\left(20 \pm 1^{\circ} \mathrm{C}\right)$ e umidade $(50 \% \pm 10 \%)$ controladas, as participantes que concordaram em participar do estudo passaram 15 minutos em espera para estabilização da temperatura adaptado de KRUEGER (2011). Foi delimitado uma área de quatro centímetros quadrados nas regiões da face denominada masseterina. Utilizando o equipamento de análise facial por bioimpedância (HTMR-W3102, MORYO, CHINA) foi realizado leve toque do sensor pelo tempo de três segundos para coleta de dados e as análises foram realizadas em triplicata. 


\section{ANÁLISE POTENCIOMETRIA (PH) FACIAL}

Em uma sala fechada com temperatura $\left(20 \pm 1^{\circ} \mathrm{C}\right)$ e umidade $(50 \% \pm 10 \%)$ adaptado de Luebberding ${ }^{9}$ controladas, as participantes que concordaram em participar do estudo passaram 15 minutos em espera para estabilização da temperatura. Foi delimitado uma área de quatro centímetros quadrados na região da face denominada masseterina. Utilizando o equipamento de análise pHmetro com sonda superficial (AMT28F, AMTAST, USA) previamente calibrado, foi borrifada na área a ser medida solução fisiológica $0,9 \%$ e com leve toque do sensor na pele por cinco segundos foi coletado o valor de $\mathrm{pH}$ para cada região.

\section{ANÁLISE DE DADOS}

O banco de dados foi criado no programa Excel - Windows $\AA^{\circledR}$ e a análise dos dados foi no Minitab 17. Foram utilizados testes estatísticos para normalidade Ryan-Joiner e Kolmogotov-Smirnov na amostra e teste T-student para analisar a diferença entre as médias.

\section{RESULTADOS / DISCUSSÃO}

A média de idade das participantes usuárias de maquiagem foi de 22,0 $( \pm 2,18)$ anos e a média idade das participantes não usuárias foi de 22,0 $( \pm 4,19)$ anos. Foram realizadas análises em triplicata de cada parâmetro $(\mathrm{pH}$, hidratação e oleosidade) da região masseteriana de pessoas usuárias e não usuárias de maquiagem, as quais os valores estão descritos na Tabela 1 e 2. 
Tabela 1. Médias e desvio padrão da hidratação, oleosidade e pH da pele de mulheres que utilizam e não utilizam maquiagem diariamente, Pernambuco, Brasil, 2019.

\begin{tabular}{|c|c|c|c|c|c|c|c|c|c|}
\hline \multicolumn{10}{|c|}{$\begin{array}{l}\text { Uso maquiagem } \\
\text { Diariamente }\end{array}$} \\
\hline & \multirow{2}{*}{\multicolumn{3}{|c|}{ Média (\%) D. P. p-Valor }} & \multicolumn{3}{|c|}{ Oleosidade } & \multicolumn{3}{|c|}{$\mathrm{pH}$} \\
\hline & & & & Média & 6) D. P & p-Valor & Médi & $\%) \mathrm{D}$. & P. p-Valor \\
\hline Sim & 33,17 & 5,20 & 0,971 & 17,90 & 2,10 & 0,903 & 5,76 & 0,46 & 0,188 \\
\hline Não & 33,12 & 3,81 & & 17,96 & 1,56 & & 5,93 & 0,49 & \\
\hline
\end{tabular}

Fonte: Elaboração Própria (2019)

A média geral de hidratação, oleosidade e pH das região da bochecha de mulheres usuárias de maquiagem foi $33,17 \%(5,20), 17,90 \%(2,10)$ e $5,76 \%(0,46)$, enquanto a média geral dos mesmos parâmetros de mulheres não usuárias foi 33,12\% $(3,81)$, $17,96 \%(1,56)$ e $5,93 \%(0,49)$. Não houve diferença significativa entres as médias das entrevistadas que relataram o uso de maquiagem diariamente e das que não fazem uso diário. Esse fato pode ter interferência pelo tamanho da amostra ou realmente por não haver diferença nos parâmetros analisados.

Os valores de $\mathrm{pH}$ da região masseteriana encontrados em nossa pesquisa foram semelhantes aos de Luebberding et al (2013), que constataram que os valores de pH são maiores na região da bochecha das mulheres ficando em torno de $5.27( \pm 0.39)$. $\mathrm{O}$ valor do $\mathrm{pH}$ é importante para a defesa cutânea contra microorganismos e no controle de enzimas epidérmicas, mas tem tendência de aumentar de acordo com a progressão da idade do indivíduo. Leonardi et al (2002), também relataram que a pele deve apresentar pH levemente ácido entre $(4,6$ - 5,8).

A média de hidratação das participantes da nossa pesquisa foi consideravelmente baixa quando comparada aos valores encontrados por Luebberding et al (2013), que avaliaram que o nível mais alto de hidratação nas mulheres é na bochecha 60,81\% $( \pm 0,81)$. Um estudo também afirmou que a oleosidade permite a manutenção e o aumento do nível de hidratação profunda da pele, evitando a perda hídrica por 
evaporação, logo a hidratação mantida na pele promove o fluxo de processos bioquímicos naturais que resultam numa pele com aspecto saudável (RAMOS-ESILVA et al., 2006).

Rangel et al. (2014) utilizaram a espectroscopia confocal de Raman para examinar a superfície da pele de voluntários. Os espectros Raman foram adquiridos antes do uso do produto e após trinta dias de uso diário de cosméticos como: ácido pirrolidona carboxílico (PCA) e o ácido urocânico (UCA). Os resultados obtidos mostraram que o uso de cosméticos faciais aumentou em $6,4 \%$ o teor de água superficial e o fator de hidratação natural da pele, esse aumento indica diminuição dos efeitos de envelhecimento cutâneo. O estudo também avaliou que uma pele normal sem aspecto de secura, apresenta teor de água maior que $10 \%$, e que a pele desidratada pode apresentar várias alterações. Todas as participantes da nossa pesquisa relataram fazer uso de algum produto cosmético, sendo a base líquida o produto mais utilizado $48,27 \%$ ( $n=28)$ e que pode influenciar nas médias de hidratação da pele das entrevistadas.

As Pesquisas de Hope (2017) apontam que a utilização diária de cosméticos por mulheres de todo mundo é de aproximadamente 06 a 12 produtos contendo de 85 a 165 ingredientes, as substâncias e os componentes ativos presentes nas formulações cosméticas podem influenciar nas propriedades mecânicas da pele. Esses ativos presentes em maquiagens não possuem apenas efeito embelezador, mas também tratam, protegem e promovem benefícios a longo prazo (LIN, 2010). 
Tabela 2 - Média e desvio padrão do tempo de exposição solar e exposição a ambientes com condicionadores de ar, Pernambuco, Brasil, 2019.

\begin{tabular}{ccccc}
\hline $\begin{array}{c}\text { Uso maquiagem } \\
\text { diariamente }\end{array}$ & \multicolumn{2}{c}{ Exposição solar } & \multicolumn{2}{c}{ Ar Condicionado } \\
Média (horas) & D.P. & Média (horas) & D.P. \\
\hline Sim & 2,27 & 1,29 & 6,06 & 4,22 \\
Não & 2,59 & 2,31 & 6,70 & 4,61 \\
& & & & \\
\hline
\end{tabular}

Fonte: Elaboração Própria (2019)

Quando questionadas sobre o tempo de exposição solar a média geral para as usuárias de maquiagem foi de 2,27 $(1,29)$ horas e ao ar-condicionado de 6,06 $(4,22)$ horas. A média geral do tempo de exposição solar das não usuárias de maquiagem foi de $2,59(2,31)$ horas e ao ar-condicionado foi de $6,70(4,61)$ horas.

Foi notado no estudo de Urasaki et al (2016) que 55\% dos jovens que participaram da pesquisa se expõem à radiação solar no horário entre 10 e 16 horas e 67,4\% ficam expostos ao sol por mais de uma hora e que $29,1 \%$ dos participantes não faziam uso de nenhum tipo de proteção solar. Os resultados foram semelhantes à nossa pesquisa que avaliou que das mulheres não usuárias de maquiagem 29,61\% nunca utilizam proteção solar. O estudo também avaliou que $50 \%$ de seus participantes só utilizavam proteção solar em dias ensolarados, enquanto $20,69 \%$ das nossas entrevistadas relataram o uso de proteção somente em exposição ao sol. Há uma relação não linear entre a exposição solar durante a vida e danos à pele e envelhecimento da pele. Portanto o tempo maior de exposição solar pode aumentar os efeitos danosos à pele (KIMLIN, 2012).

A média de horas de exposição ao ar-condicionado das participantes da nossa pesquisa foi em média de seis horas por dia. Sebastian et al (2014) analisaram os parâmetros da epiderme e mostrou que a baixa umidade relativa do ar (UR) e o clima frio em ambientes climatizados podem causar um desequilíbrio entre a pressão osmótica interna e externa da pele, provocando dessecação. O estudo de Rangel et al (2014). Mostrou que a as camadas externas da pele são hidratadas em um grau 
menor do que as mais profundas e são dependentes da umidade do ambiente. Resultados obtidos através dos métodos de espectroscopia Raman mostraram que o teor de água na superfície da pele foi de aproximadamente $30 \pm 5 \%$.

Tabela 3 - Percentual da frequência de uso do filtro solar por mulheres que utilizam e não utilizam maquiagem diariamente, Pernambuco, Brasil, 2019.

\begin{tabular}{ccccc}
\hline & \multicolumn{3}{c}{ Usuária de maquiagem diariamente } \\
\cline { 2 - 5 } Filtro solar & \multicolumn{2}{c}{ Sim } & \multicolumn{2}{c}{ Não } \\
& $\%$ & $\mathrm{n}$ & $\%$ & $\mathrm{n}$ \\
\cline { 2 - 5 } Nunca & 17,24 & 5 & 29,63 & 8 \\
Às vezes & 41,38 & 12 & 37,04 & 10 \\
Diariamente & 20,69 & 6 & 11,11 & 3 \\
Em exposição ao sol & 20,69 & 6 & 22,22 & 6 \\
Total & 100,00 & 29 & 100,00 & 27 \\
\hline
\end{tabular}

Fonte: Elaboração Própria (2019)

Das 29 mulheres usuárias de maquiagem apenas 20,69\% ( $n=6)$ fazem uso diário de filtro solar, $20,69 \%(n=6)$ relataram o uso somente em exposição ao sol, $41,38 \%$ $(n=12)$ relataram fazer uso esporádico e $17,24 \%(n=5)$ relataram nunca utilizar filtro solar. Das 27 mulheres que não utilizam maquiagem diariamente, a maioria 37,04\% $(n=10)$ relatou uso esporádico de filtro solar e apenas $11,11 \% \quad(n=3)$ utilizam diariamente, 22,22\% ( $n=6)$ utilizam apenas em exposição ao sol, e 29,63\% ( $n=8)$ nunca utilizam filtro solar.

Svobodova (2006) enfatizou que o uso de filtro solar é recomendado como estratégia primária contra danos causados pela luz solar. A eficácia dos filtros solares é tradicionalmente avaliada usando o fator de proteção solar (FPS). Assim, o FPS baseia-se na prevenção de eritema (queimadura solar) causada principalmente pelo UVB. A exposição à luz solar está relacionada ao desenvolvimento de todos os tipos de câncer de pele. Conforme os dados do Instituto Nacional de Câncer (INCA), o câncer de pele não melanoma (NMSC) corresponde a 30\% de todos os tumores malignos que são registrados no país. Urasaki et al (2016) também avaliaram a 
frequência do tipo de proteção utilizada, e certificou que as mulheres demonstraram percentagem maior e que os jovens de pele clara aderiram as mais medidas protetoras em relação aos de pele intermediária e escura. Um dos motivos seria a maior atenção à pele em decorrência dos casos de câncer na família e maior frequência de lesões por radiação (URASAKI et al., 2016).

Tabela 4. Média e desvio padrão de hidratação, oleosidade e pH por tipo de pele relatado por usuárias de maquiagens, Pernambuco, Brasil, 2019.

\begin{tabular}{ccccccc}
\hline \multirow{2}{*}{ Tipo de pele } & \multicolumn{2}{c}{ Hidratação } & \multicolumn{2}{c}{ Oleosidade } & \multicolumn{2}{c}{$\mathrm{pH}$} \\
\cline { 2 - 7 } & Média(\%) & D.P. & Média(\%) & D.P. & Média(\%) & D.P. \\
\cline { 2 - 7 } Normal & 34,48 & 0,93 & 18,48 & 0,36 & 5,73 & 0,31 \\
Oleosa & 32,21 & 5,76 & 17,37 & 2,24 & 5,79 & 0,53 \\
Mista & 34,10 & 5,15 & 18,47 & 2,13 & 5,90 & 0,26 \\
Seca & 34,09 & 0,82 & 18,12 & 0,52 & 5,82 & 0,14 \\
Sensível & 36,37 & 0,91 & 19,30 & 0,40 & 5,10 & 0,05 \\
\hline
\end{tabular}

Fonte: Elaboração Própria (2019)

Tabela 5. Média e desvio padrão de hidratação, oleosidade e pH por tipo de pele relatado por não usuárias de maquiagens, Pernambuco, Brasil.

\begin{tabular}{cccccccc}
\hline \multirow{2}{*}{ Tipo de pele } & \multicolumn{2}{c}{ Hidratação } & \multicolumn{2}{c}{ Oleosidade } & \multicolumn{2}{c}{ pH } \\
\cline { 2 - 8 } & Média(\%) & d.p. & Média(\%) & d.p. & Media & d.p. \\
\hline \multirow{2}{*}{ Normal } & 33,33 & 0,86 & 18,05 & 0,37 & 5,88 & 0,08 \\
Oleosa & 33,12 & 0,80 & 17,96 & 0,35 & 5,88 & 0,10 \\
Mista & 33,12 & 0,85 & 17,96 & 0,37 & 5,93 & 0,11 \\
\hline
\end{tabular}

Fonte: Elaboração Própria (2019)

Quando questionadas sobre o tipo de pele, o mais relatado pelas entrevistadas foi à pele sensível. $\mathrm{Na}$ tabela 4 é possível observar que os tipos de pele apresentaram 
percentual de hidratação próximos considerando o desvio padrão das análises. Os parâmetros de oleosidade e pH também apresentaram mesmo perfil. Na Tabela 5, os valores apresentaram parâmetros similares aos observados com relação a usuárias de maquiagens.

Figura 1. Problemas de pele relatados por mulheres que utilizam e não utilizam maquiagem diariamente.

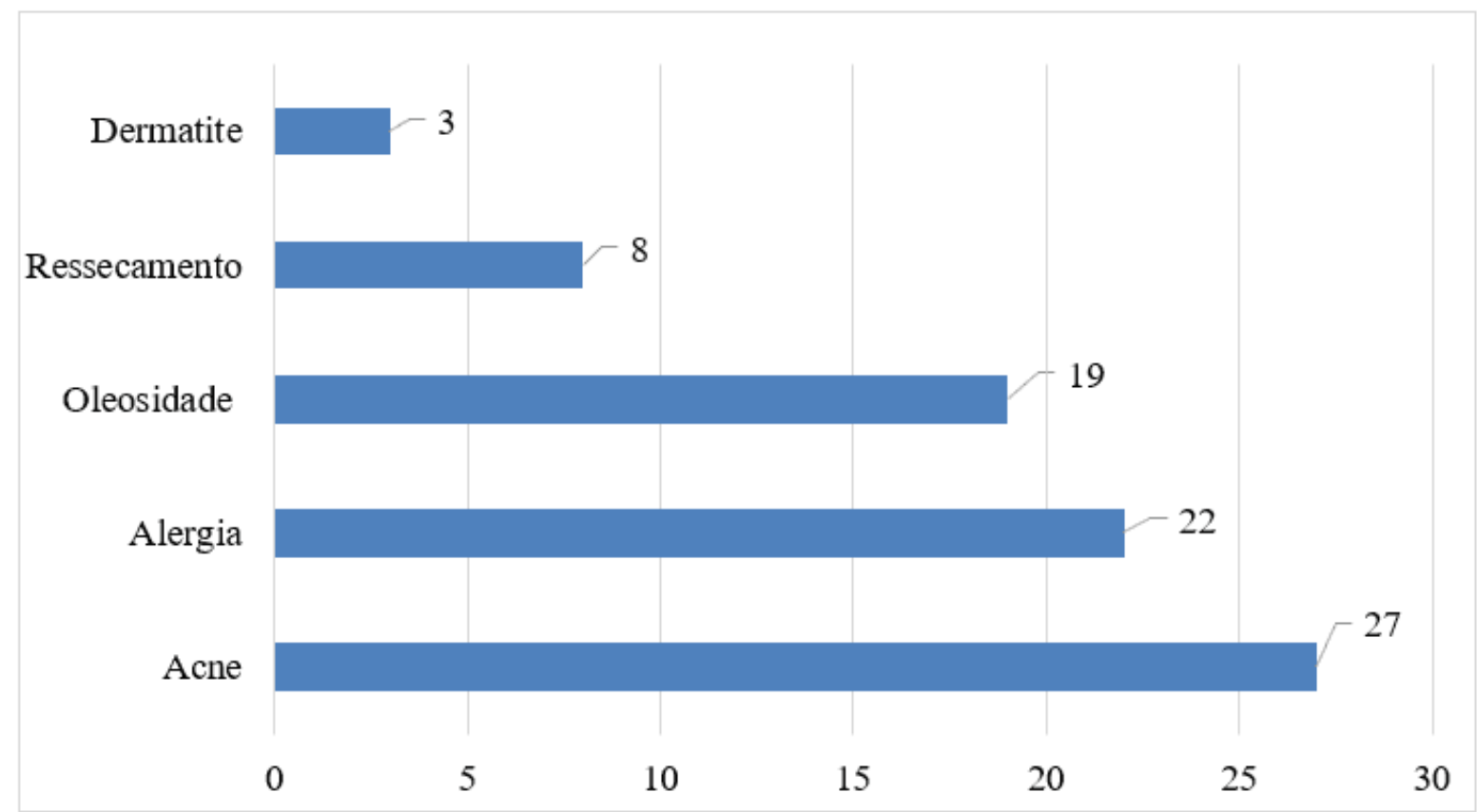

Fonte: Elaboração Própria (2019)

Quando questionadas se possuíam algum problema de pele, as entrevistadas assinalaram mais de uma vez, sendo assim, o total de respostas foi $\mathrm{N}=79$. Acne $34,17 \%(n=27)$ foi o problema relatado mais prevalente, seguido de alergias $27,84 \%$ $(n=22)$ oleosidade $24,05 \%(n=19)$, ressecemento $10,12 \%(n=8)$ e dermatites $3,79 \%$ $(n=3)$.

Tanghetti et al. (2014) descreveram as características clínicas e impacto psicossocial da acne em mulheres adultas. Das participantes da pesquisa, 40,9\% relatou que a acne foi desencadeada por cosméticos e $39,9 \%$ por maquiagens, entre outras condições. Observou-se que $58,2 \%$ das entrevistadas utilizavam maquiagem como 
medidas para lidar com a acne, e melhorar a autoestima (NASH et al 2006). Em nossa pesquisa $34,17 \%(n=27)$ das entrevistadas relataram ter acne. Os fatores que contribuem para formação de acne são induzidos pela produção de sebo, inflamação, propionibacterium e colonização do fólico piloso (LIU et al., 2016). Matsuoka et al (2006) avaliaram que $80 \%$ das entrevistadas com acne, em sua rotina diária usou produtos de cuidados com a pele e maquiagem, constatou-se que o uso de maquiagem sob instrução de dermatologistas pode ter um efeito favorável sobre as condições psicológicas ou relações interpessoais melhorando a autoestima (CAISEY et al., 2006). Algumas formulações de base corretiva podem conter pigmentos, conservantes, metais, parabenos, fragrâncias, tensoativos entre outras substâncias que estão associados à alergia, aparecimento de acne e dermatites (CHORILLI et al., 2007). Sendo esses os mesmos problemas relatados pelas entrevistadas da nossa pesquisa.

Figura 2. Descrição dos agentes de limpeza empregados na rotina de higienização da pele por mulheres que utilizam e não utilizam maquiagem diariamente.

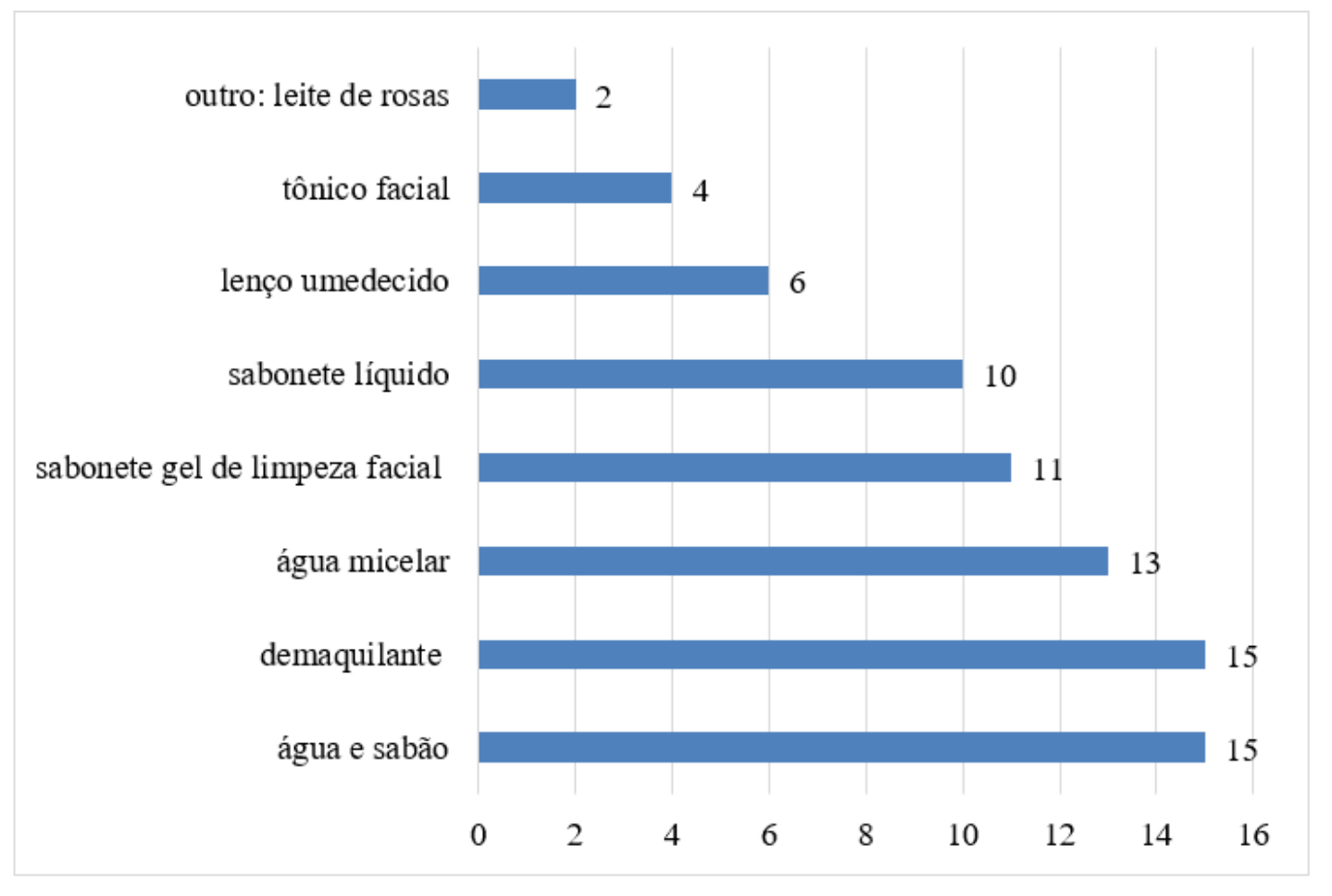

Fonte: Elaboração Própria (2019) 
Quando questionadas sobre os tipos de agentes de limpeza mais utilizados nos cuidados com a pele as entrevistadas assinalaram mais de uma vez, o total de respostas foi $\mathrm{N}=76$. Os agentes mais utilizados foram água e sabão $19,73 \%(n=15)$ e demaquilante $19,73 \%$ ( $n=15)$, seguidos de água micelar $17,10 \%(n=13)$, sabonete em gel de limpeza facial $14,47 \%(n=11)$, sabonete líquido $13,15 \% \quad(n=10)$, lenço umedecido $7,89 \%(n=6)$, tônico facial $5,26 \%(n=4)$ e leite de rosas $2,63 \%(n=2)$.

O agente de limpeza mais utilizado pelas nossas participantes foi água e sabão. Segundo o estudo de Mahmud (2015), a limpeza da pele significa remover impurezas (incluindo bactérias) da superfície da pele, a partir da atmosfera ou da aplicação de produtos cosméticos. É importante remover os subprodutos indesejáveis criados por microrganismos para obter limpeza e frescura. O estudo também relata que a limpeza da pele pode influenciar as alterações de $\mathrm{pH}$ da superfície da pele. Com base $\mathrm{no} \mathrm{pH}$ do limpador e os produtos de limpeza ácidos são menos irritantes em comparação com produtos de limpeza alcalinos e neutros. A água pura ( $\mathrm{pH}$ na faixa de 6,5 a 9,5) também pode ter um impacto no valor do $\mathrm{pH}$ da pele.

Matsumoto et al (2014), avaliaram diferentes métodos de lavagem da pele, os método de lavagem com produtos protetores e não protetores, foi analisado os parâmetros da pele através dos métodos de bioengenharia, as análises foram realizadas em triplicata em um laboratório com ambiente na temperatura constante de $25^{\circ} \mathrm{C}$ e umidade de $50^{\circ} \mathrm{C}$. $\mathrm{O}$ estudo mostrou que $\mathrm{opH}$ teve um aumento significativo na pré- lavagem $(P<0,05)$ lavando com produtos protetor do que com produtos não protetores, a fricção da pele durante a lavagem exerce um estímulo mecanico na pele que pode causar alterações na textura da pele ocasionando vermelhidão. 
Figura 3. Descrição dos tipos de produtos faciais mais utilizados empregados na rotina de cuidados da pele por mulheres que utilizam e não utilizam maquiagem diariamente.

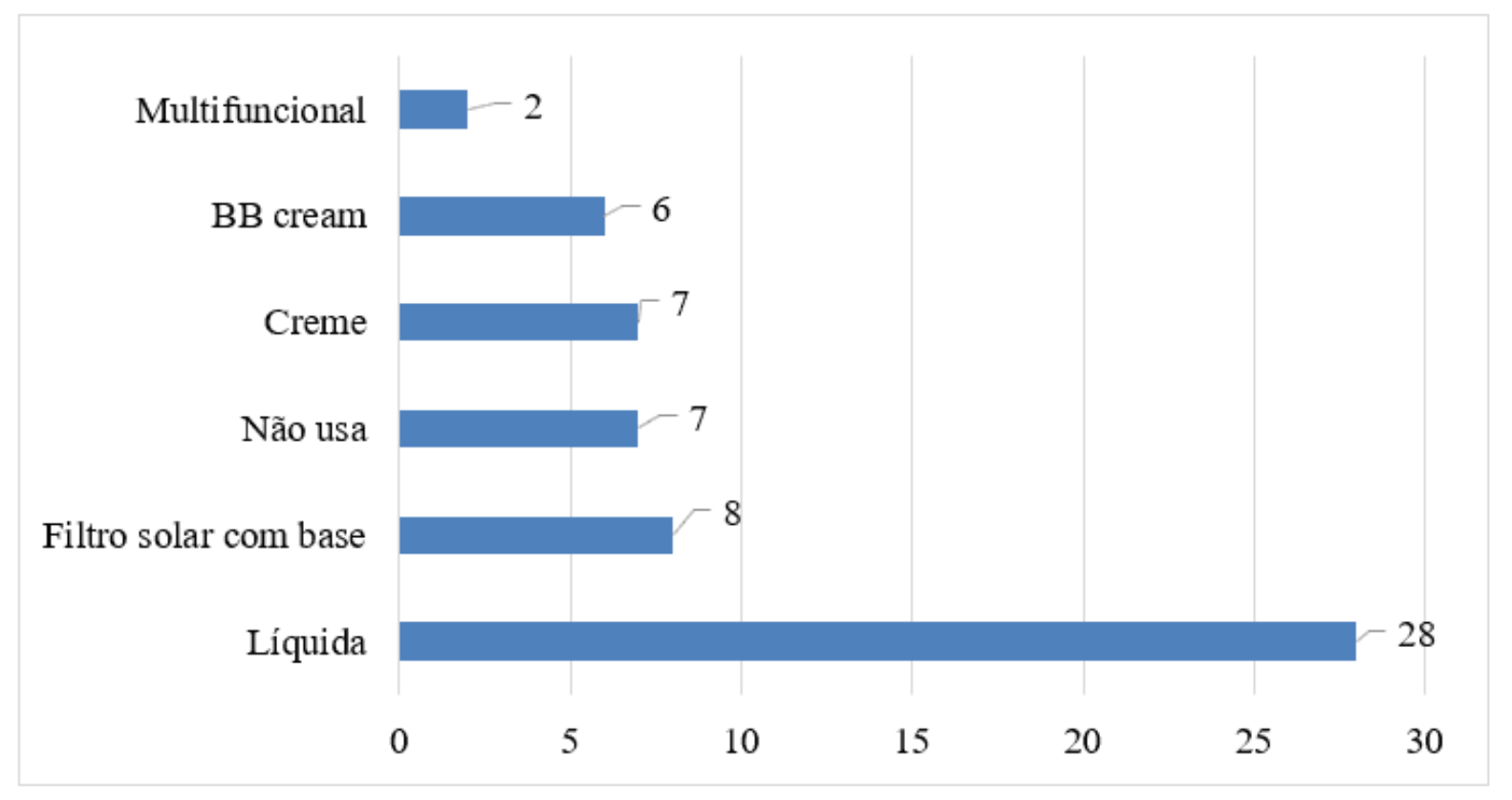

Fonte: Elaboração própria (2019)

As entrevistadas assinalaram sobre o tipo de produto facial mais utilizado na rotina de cuidados com a pele, o total de respostas foi $\mathrm{N}=58$. Base líquida $48,27 \%(n=28)$ foi o produto facial mais utilizado pelas mulheres entrevistadas, seguida da base corretiva com filtro solar $13,79 \%(n=8)$, creme $12,06 \%(n=7)$, BB cream $10,34 \%(n=6)$, Multifuncional $3,44 \%(n=2)$. Algumas entrevistadas relataram não fazer uso de produtos faciais, sendo $12,06 \%(n=7)$.

Um estudo revelou que as reações adversas a cosméticos podem ser irritativas, alérgicas ou sensibilizantes, dermatite por fotossensibilidade, reações sistêmicas, entre outras. A irritação que pode ser definida como intolerância local podendo corresponder a reações de desconforto variando sua intensidade desde a dor, coceira e punicação podendo chegar até a corrosão e destruição do tecido. Todas essas reações se restringem à área em contato direto com o produto (CHORILLI et al., 2007). Em nossa pesquisa as entrevistadas também relataram sofrer com alergias 27,84\% $(n=22)$ oleosidade $24,05 \%(n=19)$, ressecamento $10,12 \%(n=8)$ e dermatites $3,79 \%$ $(n=3)$ 
Um estudo publicado por Choi; Choi; Youn (2012) mostra que para fins de cosméticos a pele facial é classificada em diferentes tipos de pele. Os fatores intrínsecos e extrínsecos são responsáveis pelo tipo de pele. Visto que esses fatores podem prejudicar o manto hidrofílico que mantém água na superfície da pele, e na produção de sebo que está a associado a suavidade e maciez na pele podendo desencadear ressecamento e oleosidade na pele (SAKUMA, 2012).

\section{CONSIDERAÇOES FINAIS}

Conforme os resultados obtidos na pesquisa, não houve diferenças significativas nos valores de hidratação, $\mathrm{pH}$ e oleosidade na comparação da pele de mulheres usuárias e não usuárias de maquiagem.

Compreende-se que as variações da cor e as características da pele em diferentes grupos étnicos influenciam no desenvolvimento de linhas de produtos faciais. Considera-se que os tipos de produtos cosméticos utilizados para a limpeza da pele e os tipos de base corretiva podem estar relacionados com o relato dos principais problemas de pele relatados pelas participantes dessa pesquisa.

Evidencia-se que as reações adversas a cosméticos podem ser ocasionadas principalmente pelos conservantes e metais aplicados na formulação desses produtos. As formulações cosméticas precisam apresentar segurança, biocompatibilidade e funcionalidade. Conclui-se que é primordial o desenvolvimento de testes que comprovem o alto nível de segurança do produto o qual poderá ser utilizado por consumidores de vários tipos e condições de pele.

\section{REFERÊNCIAS}

BENJAMIN, Helen et al. A planar micro-sensor for bio-impedance measurements. SensorsAnd Actuators B: Chemical, [s.I.], v. 111-112, p.430-435, nov. 2005.

CAISEY, L. et al. Skin color and makeup strategies of women from different ethnicgroups. International Journal Of Cosmetic Science. France, p. 427-437. mar. 2006. 
CHORILLI, Marlus et al. Toxicologia dos Cosméticos. Latin American Journal Of Pharmacy(formerlyacta Farmacéutica Bonaerense. São Paulo, p. 144-54. ago. 2007.

CHOI, C. W.; CHOI, J. W.; YOUN, S. W.. Subjective facial skin type, based on the sebum related symptoms, can reflect the objective casual sebum level in acne patients. Skin Research And Technology, [s.I.], v. 19, n. 2, p.176-182, 29 dez. 2012. Wiley.

CONSTANTIN, Maria-magdalena et al. Skin Hydration Assessment through ModernNon- Invasive Bioengineering Technologies. Maedica (buchar). S.i, p. 33-38. mar. 2014.

CHOI, C. W.; CHOI, J. W.; YOUN, S. W.. Subjective facial skin type, based on the sebum related symptoms, can reflect the objective casual sebum level in acne patients. Skin Research And Technology, [s.I.], v. 19, n. 2, p.176-182, 29 dez. 2012. Wiley.

FIROOZ, Alireza et al. Daytime Changes of Skin Biophysical Characteristics: A Study of Hydration, Transepidermal Water Loss, pH, Sebum, Elasticity, Erythema, and Color Index on Middle Eastern Skin. Indian J Dermatol, Índia, v. 3, n. 61, p.61-700,set.2016.

HOPPE, Ana Carolina; PAIS, Mariana Castello Novo. Avaliação da toxicidade de parabenos em cosméticos. Revinter, Brasil, v. 10, n. 3, p.49-70, out. 2017.

HUANG, Xian et al. Epidermal Differential Impedance Sensor for Conformal Skin Hydration Monitoring. Biointerphases, S.i, p.7-52, 23 jul. 2012.

IGWO-EZIKPE, Miriam N. et al. Heavy metals and Polycyclic Aromatic Hydrocarbons in Some Facial Makeup Cosmetics Commonly Used in Lagos, Nigeria. Nigerian Society For Experimental Biology (, Nigerian, v. 29, n. 03, p.110-125, 14 out. 2017. 
KIMLIN, Michael G.; GUO, Yuming.Assessing the impacts of lifetime sun exposure on skin damage and skin aging using a non-invasive method. Science Of The Total Environment, [s.I.], v. 425, p.35-41, maio 2012. Elsevier BV.

KRUEGER, Nils et al. Age-related changes in skin mechanical properties: a quantitative evaluation of 120 female subjects. Skin Research And Technology,[s.I.], v. 17, n. 2, p.141-148, 1 fev. 2011.

LEONARDI, Gislaine Ricci; GASPAR2, Lorena Rigo; CAMPOS, Patrícia M. B. G. Maia. Study of $\mathrm{pH}$ variation on the skin using cosmetic formulation $\mathrm{s}$ with and without vitamins A, E or ceramide: by a non-invasive method. Anais Brasileiros de Dermatologia, Rio de Janeiro, v. 5, n. 77, p.563-569, out. 2002. 563-569, set./out. 2002.

LIN, T. Joseph. Evolution of Cosmetics: Increased Need for Experimental ClinicalMedicine. Journal Of Experimental \& Clinical Medicine, [s.I.], v. 2, n. 2, p.52,abr.2010.

LUEBBERDING, S.; KRUEGER, N.; KERSCHER, M.. Skin physiology in men and women: in vivoevaluation of 300 people including TEWL, SC hydration, sebum content and skin surface $\mathrm{pH}$. International Journal Of Cosmetic Science, [s.I.], v. 35, n. 5, p.477-483, 6 jul. 2013.

MAHMUD, Tunku Hana Tunku; ABDUL-AZIZ, Azila; MUDA, Rohaiza. A Review on the Potential Use of Chitosan-Based Delivery System in Mild Facial Cleansing Formulation. International Journal Of Polymeric Materials And Polymeric Biomaterials, [s.I.], v. 64, n. 8, p.432-437, 6 jan. 2015.

MONTEIRO, Bruna Elisabete Silva. Toxicidade dos produtos cosméticos. 2017. 83 f. Monografia (Especialização) - Curso de Farmácia, Universidade Fernando Pessoa, Porto,2017. Cap. 83.

MATSUOKA, Yoshie et al. Effects of skin care and makeup under instructions from dermatologists on the quality of life of female patients with acne vulgaris. The Journal Of Dermatology, [s.I.], v. 33, n. 11, p.745-752, nov. 2006. Wiley. 
MATSUMOTO, M. et al. Influence of differences in washing methods on skin text. International Journal Of Cosmetic Science,. S.i, p. 175-181. jan. 2014.

NASH, Rebecca et al. Cosmetics: They Influence More Than Caucasian Female Facial Attractiveness. Journal Of Applied Social Psychology, [s.I.], v. 36, n. 2, p.493-504, 30 mar.2006. Wiley.

RAMOS-E-SILVA, Marcia et al. Journal of Cosmetic Dermatology. Blackwell Publishing. Rio de Janeiro, p. 40-50. 15 jul. 2006.

RANGEL, Ana Paula et al. Confocal Raman spectroscopy: determination of natural moisturizing factor profile related to skin hydration. Revista Brasileira de Engenharia Biomédica, [s.I.], v. 30, n. 1, p.11-16, 2014. EditoraCuboMultimidia.

RYU, Hyo Sub et al. Influence of age and regional differences on skin elasticity as measured by the Cutometer. JournalCompilation. S.i, p. 354-358. dez. 2008.

SAKUMA, T Hais H.; MAIBACH, Howard I.. Oily Skin: An Overview. S KinPharmacolPhysiol, An Francisco, Calif., Usa, n. 25, p.227-235, 20 jun. 2012.

SEBASTIAN et al Stratum corneum molecular mobility in the presence of natural moisturizers. This Journal Is (c) The Royal Society Of Chemistry: , 10, 45354546. S.i, p. 4535-4546. abr. 2014.

SCHNITTGER, Steven; SINHA, Moitreyee.The Materials Science of Cosmetics. MBulletin, [s.I.], v. 32, n. 10, p.760-769, out. 2007. Cambridge University Press (CUP).

SVOBODOVA, Alena; WALTEROVA, Daniela; VOSTALOVA, Jitka. ULTRAVIOLET LIGHT INDUCED ALTERATION TO THE SKIN. Biomed Pap Med Fac Univ Palacky Olomouc Czech Repub, S.i, v. 1, n. 150, p.25-38, maio 2006.

TANGHETTI, Emil A. et al. Entendendo o ônus da acne feminina adulta. J Clin Aesthet Dermatol. S.i, p. 22-30. fev. 2014. 
URASAKI, Maristela Belletti Mutt et al. Práticas de exposição e proteção solar de jovens universitários. Revista Brasileira de Enfermagem, [s.I.], v. 69, n. 1, p.126133, fev. 2016. FapUNIFESP (SciELO).

\section{ANEXO}

\section{Anexo I - Questionário adaptado}

QUESTIONÁRIO PARA COLETA DE DADOS SOBRE A UTILIZAÇÃO DE MAQUIAGEM DO TIPO BASE CORRETIVA DE MULHERES USUÁRIA E NÃO USUÁRIA DE MAQUIAGEM. (Adaptado de KIMLIN, 2012)

PERFIL SÓCIO DEMOGRÁFICO:

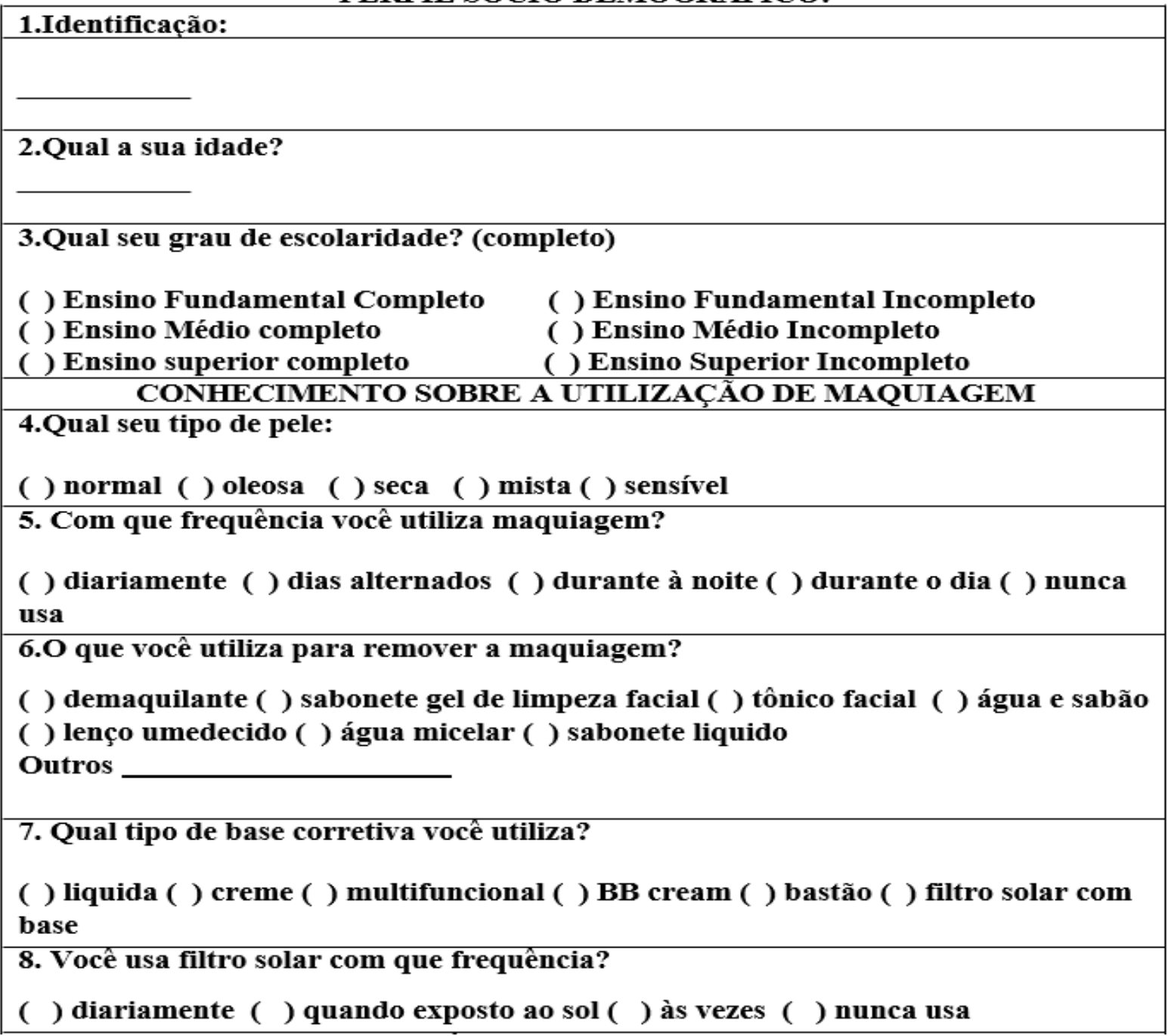


9.Por quantas horas por dia você se expõe à radiação solar?

10. Por quantas horas por dia você se expõe ao ar-condicionado?

11. Você já teve algum problema na pele com a utilização de maquiagem?

( ) dermatites ( ) acne ( ) oleosidade ( ) ressecamento ( ) alergia

Enviado: Junho, 2020.

Aprovado: Janeiro, 2021. 